\title{
Inteligencia emocional y su relación con las estrategias de afrontamiento al estrés en alumnos de Pachuca de Soto, Hidalgo
}

\section{Emotional intelligence and its relationship with stress coping strategies in students from Pachuca de Soto, Hidalgo}

Rangel-Pagola Carlos Alejandro ${ }^{a}$

\begin{abstract}
:
This article was designed to analyze emotional intelligence and its relationship with stress coping strategies which were analyzed in the SPSS data processor, the research was carried out based on quantitative methodology, in order to carry out the development of the objectives, had a correlational-causal design; The sample consisted of 384 students from high school No. 1, through a nonprobabilistic sample, because not all had the same probability of participating, to evaluate the variables respectively, the BarOn Emotional Intelligence Inventory was applied: version for young people (7-18 years) and the Assessment Instrument for Stress Coping Strategies; The statistical procedure was the Spearman Rho correlation, in the results it is revealed that there is a significant correlation in the strategies focused on problems, yielding a score of .36 (<.01), as well as the strategies focused on emotions with a level of .41 $(\mathrm{p}<.01)$, on the other hand, a non-significant correlation is found in strategies focused on avoidance with a correlation of .16 ( $p<.01)$. It is proposed in future research to develop studies with larger samples to obtain significant statistical ranges.
\end{abstract}

Keywords:

stress coping strategies, emotional intelligence, preparatory

Resumen:

El presente artículo fue diseñado para analizar la inteligencia emocional y su relación con las estrategias de afrontamiento al estrés los cuales fueron analizados en el procesador de datos SPSS, la investigacion se realizó en base a la metodología cuantitativa, para poder llevar acabo el desarrollo de los objetivos, tuvo un diseño correlacional-causal; la muestra estuvo compuesta por 384 alumnos de la preparatoria No. 1, mediante una muestra no probabilística, debido a que no todos tenían la misma probabilidad de participar, para evaluar las variables respectivamente se aplicó el Inventario de Inteligencia Emocional de BarOn: versión para jóvenes (7-18 años) y el Instrumento de evaluación para estrategias de afrontamiento al estrés; el procedimiento estadístico fue la correlación Rho de Spearman, en los resultados se revela que existe una correlación significativa en las estrategias centradas en los problemas arrojando una puntuación de $.36(<.01)$, al igual que las estrategias centradas en las emociones con un nivel de $.41(\mathrm{p}<.01)$, por otra parte, se encuentra una correlación no significativa en las estrategias centradas en la evitación con una correlación de .16 ( $\mathrm{p}<.01$ ). Se propone en fututas investigaciones desarrollar estudios con muestras mayores para obtener rangos estadísticos significativos.

Palabras Clave:

estrategias de afrontamiento al estrés, inteligencia emocional, preparatoria

\section{Introducción}

La inteligencia emocional es una habilidad necesaria para el buen funcionamiento de la persona en cuanto a mejorar las relaciones interpersonales, uno de los pioneros en el tema como Daniel Goleman (1995), lo refiere como un conjunto de destrezas, actitudes, habilidades y competencias que determinan la conducta del individuo, por otra parte Bar-On (1997), define a la inteligencia emocional como una serie de capacidades y habilidades que se adquieren a nivel cognitivo y son influenciados por la motivación, de tal manera que permite un medio ambiente exitoso; con posteriores estudios (Gómez et al., 2000), menciona que engloba una serie de impulsos, la motivación y el entusiasmo generan una forma de actuar e interactuar con el mundo, tomando en cuenta los sentimientos y habilidades personales e interpersonales. De este modo, se infiere que la inteligencia emocional incluye una serie de características 
que implica poder adaptarse al contexto respondiendo de manera adecuada directamente sobre las emociones favoreciendo las relaciones interpersonales. Goleman (1996), explico un conjunto de dimensiones que se contemplan y son estructuradas por aptitudes emocionales y dividida en cinco dimensiones:

1. Autoconocimiento emocional: Reconocer los sentimientos mientras se experimentan, son piezas claves para tener una vida satisfactoria, básicamente la inteligencia emocional se basa en la capacidad de reconocer los sentimientos ajenos y propios;

2. Autorregulación: Capacidad de hacer frente a nuestros propios sentimientos y adaptarlos a cualquier situación, quienes carecen de esta característica son propensos a tener estados de inseguridad, y, por el contrario, quienes hacen uso adecuado tienden a recuperarse más rapido con contratiempos ocasionados por el contexto;

3. Motivación: Para Ander-Egg (1991), es aquella capacidad que tiene el ser humano para dirigir la energía a un punto especifico con un propósito explicito, se utiliza para mantener el sistema en funcionamiento, desde esta perspectiva;

4. Empatía o el reconocimiento de las emociones ajenas: La empatía es la habilidad que permite a las personas reconocer las necesidades y deseos de otros, aquellos quienes tienen esta habilidad desarrollada son más atentas y tienden a saber escuchar a los demas, comprendiendo y brindando ayuda a las necesidades, sentimientos y acciones de los demas;

5. Habilidades sociales: Es el arte de relacionarse con los demas favoreciendo las relaciones interpersonales, produciendo sentimientos hacia los demas, esta habilidad se basa en que las personas sean más eficientes en su interacción entre individuos, cabe destacar la importancia del ser asertivo y tener una escucha activa en esta dimensión.

Por otra parte, estudios recientes como Bar-On y Parker (2018), clasifican la inteligencia emocional en cuatro áreas

1. Intrapersonal: Es el autoconocimiento y autoexpresión emocional de uno mismo;

2. Interpersonal: Capacidad para tener relaciones satisfactorias con otras personas;

3. Adaptabilidad: Capacidad para gestionar el cambio;

4. Manejo del estrés: Capacidad para el manejo y la autorregulación de las emociones en diferentes situaciones estresantes.

La transición a la vida adulta trae consigo una serie de cambios tanto físicos como psicológicos, el campo de la psicologia se ha enfocado en bridar informacion oportuna y de manera holística sobre temas de desarrollo, no obstante estamos frente a una situación donde las demandas sociales exigen una cantidad de ocupaciones que pueden ir degenerando al individuo de manera biopsicosocial, es importante considerar aquellos aspectos que pueden verse vulnerables ante dichas situaciones, aquellas exigen una carga estresante importantes y afecta de manera directa la salud mental, Lazarus y Folkman (1984), citados por Beltrao (2016), definen al afrontamiento como acciones (cognitivas) 0 comportamiento (conductuales) que se modifican según las necesidades, para calificar, tolerar, modificar y cuestionar necesidades internas como externas especificas hacia la sobrecarga; por otra parte Camilo y Páez (2013), hacen referencia al resultado de un individuo para adaptarse exitosamente a las consecuencias y circunstancias del momento desafiante, favoreciendo la salud mental y vida en general, actualmente reviven el concepto de aquellas características que posee una persona para producir esfuerzos a realizar actividades 0 acciones a situaciones que se asumen como demandantes, de esta manera depende de características personales y sobre todo una evaluación a la situación que se vive en ese momento, con un repertorio de recursos o experiencias que posea el individuo; de este modo Collas y Cuzcano (2019), afirman que, en una sociedad donde se exige leer o escribir es considerado importante debido al impacto que tiene la vida laboral y de este modo, el poder adaptarse requiere de grandes esfuerzos tanto cognitivos y conductuales, alguno de los obstáculos que se pueden vivir es la angustia o la ansiedad y con esto, traer consecuencias al individuo; todos los individuos centran todos sus problemas a buscar soluciones y esfuerzos para remediar la situación mediante estrategias y formas racionales. Rojas (2018), menciona que existen estilos para hacer frente a situaciones y son estos mismos quienes determinan la estabilidad temporal y situacional, de este modo, las estrategias de afrontamiento son los procesos que se utilizan en las situaciones en cada contexto o situación, dependen y pueden cambiar dependiendo las acciones que ejecute el individuo, los estudios realizados por Frydenberg \&Lewis en (1997), se encuentran centrados en el afrontamiento en adolescentes; hoy en día, las estrategias de afrontamiento se clasifican de la siguiente manera:

1. Dirigidos al problema: Son conductas centradas en hacerle frente al problema en cuestión, es decir, ataca el origen y busca eliminar toda emoción existente, las acciones son más racionales;

2. Las estrategias centradas en la emoción, buscan solucionar y remediar la situación, mediante experiencias vividas y en la regulación directa de 
las emociones, tienden a ser más pasiva y a escapar de la situación;

3. Centradas en la evitación: Se hace lo posible por evitar, posponer o saltar la acción sin ejercer ningún tipo de desgaste emocional.

Lazarus y Folkman (1984), citados por Gaete (2015), consideran que las respuestas de afrontamiento dependen de la manera en que las personas actúan ante el estrés, $y$, por lo tanto, se ejecutan de dos maneras:

1. La función a la solución de problemas implica situaciones internas como externas, es decir, pueden suponer y descompensar la relacion que tiene el individuo con el contexto, del mismo modo, pueden aportar nuevas maneras de adaptación según sea el caso y/o las condiciones ambientales;

2. La función a la situación de problemas, incluye esfuerzos para adaptarse al malestar en la cuestión emocional provocado por eventos y acontecimientos estresantes, es decir, el individuo puede conseguir la satisfacción evitando el problema o atendiendo a manera positiva a la cuestión.

Las dos funciones estan conectadas, de cierta manera una conducta puede tener diferentes funciones, de este modo, si una situación se encuentra enfocada a la solución de problemas puede aportar y contribuir al manejo de emociones, la mayoría de las personas utilizan las estrategias de afrontamiento y cumplen estas dos funciones simultáneamente (Frydenberg \& Lewis 1997). El presente artículo de investigacion tiene como finalidad analizar la inteligencia emocional y su relacion con las estrategias de afrontamiento al estrés utilizada por alumnos de la preparatoria No. 1 ubicada en la ciudad de Pachuca de Soto, Hidalgo.

\section{Método}

Se realizó un diseño de investigacion correlacional-causal, para la obtención de la muestra se obtuvo por medio de la estadistica con un nivel de confianza del $95 \%$ y un margen de error de $5 \%$, en el estado de Hidalgo según datos arrojados de la Dirección General de Información y Estadistica Educativa (2019), existe una población de 147,365 alumnos a nivel media superior, y por su parte la Universidad Autónoma del Estado de Hidalgo (2019), cuenta con una población de 59,369 alumnos de nivel medio superior, para la recolección de datos se encuestaron 384 alumnos de la preparatoria No. 1, para alcanzar los objetivos de la presente investigación se seleccionó una muestra no probabilística, debido a que no todos tienen la misma probabilidad de participar (Hernández, Fernández y
Baptista, 2014). Los adolescentes fueron seleccionados en base a una serie de criterios:

Criterios de inclusión:

1. Ser estudiante de la UAEH

2. Ser estudiante matriculado a la Escuela Preparatoria No. 1.

Criterios de exclusión:

1. Ser estudiante de cualquier otra preparatoria. Criterios de eliminación:

1. No contestar a todos los ítems de los instrumentos.

Para el desarrollo de este estudio se emplearon distintos instrumentos para sus respectivas variables, se elaboró un instrumento fundamentado en la teoría de Frydenberg y Lewis (1997), en la cual se consideran tres tipos de estrategia: enfocados al problema, a las emociones y a la evitación; en el análisis estadístico de este inventario los ítems que evalúan las estrategias de afrontamiento enfocadas en el problema obtuvieron un Alfa de Cronbach de .630 , los ítems relacionados a las estrategias de afrontamiento enfocadas en las emociones lograron un Alfa de Cronbach de .673, por ultimo los ítems de las estrategias enfocadas en la evitación arrojan una puntuación Alfa de Cronbach de .715, esto indica que el instrumento es confiable.

El siguiente instrumento que se utilizo fue el Inventario de Inteligencia Emocional de BarOn: versión para jóvenes (7-18 años) con la finalidad de evaluar la inteligencia emocional en sus diferentes componentes socioemocionales (intrapersonal, interpersonal, adaptabilidad y manejo de estrés) (Bar-On y Parker, 2018).

La primera etapa del procedimiento fue el acercamiento con los adolescentes, se procedió a realizar la invitación con el propósito de informar los objetivos del proyecto de investigación y de manera subsecuente se proporcionaron los cuestionarios a través de la plataforma Google Forms, el periodo de evaluación fue durante los meses agosto a noviembre del 2020.

Para comprobar las hipótesis propuestas en la presente investigacion, se realizó un análisis estadístico por medio de la prueba coeficiente de correlación Rho de Spearman, para llevar a cabo el análisis se hizo uso del programa estadístico IBM Statistical Package for the Social Sciences (SPSS) versión 25. 


\section{Resultados}

\section{Tabla 1}

Distribución de frecuencias de la variable edad y su relación con la variable sexo.

Dentro de las variables demográficas se encontró

\begin{tabular}{ccccc}
\hline & & Femenino & Masculino & Total \\
\hline Edad & 16 & 56 & 46 & 102 \\
& 17 & 122 & 68 & 190 \\
& 18 & 31 & 61 & 92 \\
\hline Total & & 209 & 175 & 384
\end{tabular}

lo siguiente: $102(26.6 \%)$ sujetos se encuentran entre los 16 años, mientras que 190 (49.5\%) tienen 17 años y por último tan solo $92(24.0 \%)$ tienen 18 años de edad; respecto al sexo hay una diferencia de 209 (54.4) mujeres y $175(45.6 \%)$ hombres.

\section{Figura 1}

Distribución de frecuencias de la variable edad y su relación con la variable sexo.

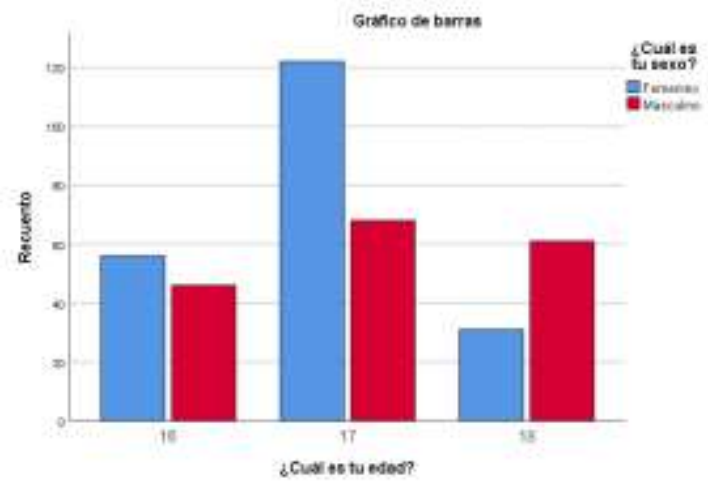

Nota: Se puede observar en la figura 1, la distribución correspondiente de cada sexo en relación con su edad.
Tabla 2

Distribución de frecuencias según las estrategias de afrontamiento utilizada por los alumnos.

\begin{tabular}{cccc}
\hline & Femenino & Masculino & Total \\
\hline $\begin{array}{c}\text { Centrados en los } \\
\text { problemas }\end{array}$ & 91 & 105 & 196 \\
$\begin{array}{c}\text { Centrados en las } \\
\text { emociones } \\
\begin{array}{c}\text { Centrados en la } \\
\text { evitación }\end{array}\end{array}$ & 83 & 30 & 113 \\
\hline Total & 217 & 167 & 384
\end{tabular}

Tras analizar las distribuciones de frecuencias y los porcentajes obtenidos se puede observar en la tabla 2 , que las estrategias enfocadas en el problema obtuvieron un porcentaje de $51.04 \%$, mientras que las estrategias enfocadas en las emociones arrojan un porcentaje de $29.43 \%$ y por ultimo las estrategias enfocadas en la evitación muestran un porcentaje de $19.53 \%$.

\section{Figura 2}

Distribución de frecuencias según las estrategias de afrontamiento utilizada por los alumnos.

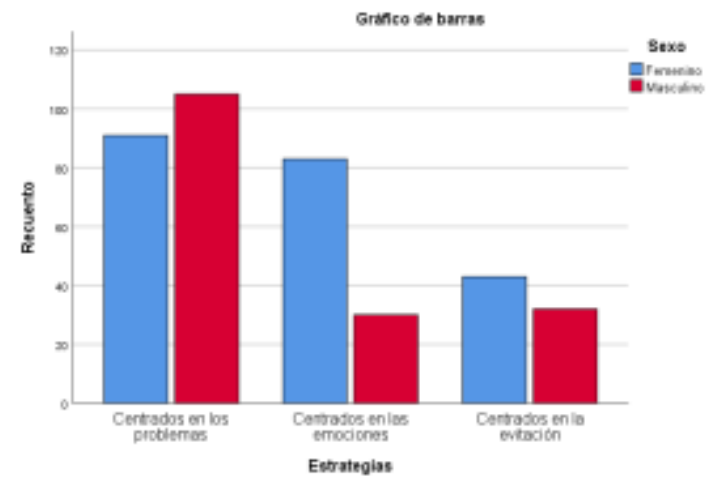

Por otra parte, en la figura 2 se observa la variable sexo con las estrategias de afrontamiento respectivamente utilizadas por los alumnos, se puede observar que los varones ocupan más las estrategias de afrontamiento centrado en los problemas con un porcentaje de $53.57 \%$ mientras que las mujeres ocupan un $46.43 \%$, respecto a las estrategias de afrontamiento centradas en las emociones existe una gran diferencia entre los hombres que ocupan un $26.5 \%$ mientras que las mujeres hacen uso del $73.5 \%$, por último en las estrategias de afrontamiento centrado en la evitación no se observa diferencia significativa entre mujeres que 
ocupan un $42.7 \%$ a diferencia de los hombres que ocupan un $57.3 \%$.

Una vez procesados los datos, se procedió analizar la correlación entre la inteligencia emocional con las estrategias de afrontamiento al estrés en donde se observa una correlación entre la inteligencia emocional y las estrategias de afrontamiento al estrés centradas en los problemas, existe una correlación entre ambas variables siendo de .43 ( $p<.01$ ) lo cual indica que los alumnos suelen utilizar con mayor frecuencia estas habilidades, mientras tanto la correlación arrojada a las estrategias centrada en las emociones es de .36 ( $p<.01)$, este resultado permite conocer que los alumnos pueden ocupar de igual manera este tipo de habilidad para enfrentar situaciones estresantes, por ultimo las estrategias centradas en la evitación muestra una correlación de .16 $(p<.01)$ lo cual indica una correlación no significativa entre ambas variables.

\section{Discusión}

De acuerdo con lo que se esperaba, los resultados de la presente investigacion sugieren que los adolescentes presentan relación entre ambas variables, lo cual se aprueba la hipótesis que se habia planteado para la investigacion, en la que se planea la existencia de un vínculo entre los componentes de la inteligencia emocional y los estilos de afrontamiento al estrés, por último, se consideran que en posibles líneas de investigación seria importante ampliar la población y aplicarlo a distintas edades.

\section{Conclusiones}

De acuerdo a los resultados que se obtuvieron se concluye lo siguiente:

1. Existe relacion entre las variables de inteligencia emocional y estrategias de afrontamiento al estrés, centrados en los problemas y en las emociones.

2. No existe relacion significativa entre la inteligencia emocional y las estrategias de afrontamiento al estrés centradas en la evitación.

3. Se propone en fututas investigaciones desarrollar estudios con muestras mayores para obtener rangos estadísticos significativos.

\section{Referencias}

Ander-Egg, E. (1991). Teoría Motivacional. McGraw-Hill Education.

Bar-On, R. (1997). The Emotional Quotient inventory (EQ-I): Technical Manual. Multi-Health Systems.

Bar-On, R., \& Parker, J. D. (2018). Inventario de Inteligencia Emocional de BarOn: versión para jóvenes (R. Barmejo, C. Ferrándiz, M. Ferrando, M. D. Prieto y M. Sáinz, adaptadoras). TEA Ediciones.

Beltrão Tavares, Livânia (2016). Estrategias de afrontamiento frente al diagnóstico de dislexia en adolescentes diagnosticados en esta fase. Subjetividad y Procesos Cognitivos, 20 (1), 38-56.

https://www.redalyc.org/articulo.oa?id=339646009002

Camilo, J., \& Páez, E. (2013). La resiliencia y el afrontamiento positivo: conceptos atados. Boletín informativo $N^{\circ} 3$ Caminos para la resiliencia, 1-3.

Collas, D. H., \& Cuzcano, S. D. (2019). Estrategias de afrontamiento ante el estrés y bienestar psicológico en estudiantes universitarios de una institución privada de Lima Este, 2018. Universidad Peruana Unión.

Dirección general de información y estadística educativa. (2019). Publicación estadística educativa. SEP.

Frydenberg, E., \& Lewis, R. (1997). ACS Escalas de afrontamiento para adolescentes. TEA Ediciones.

Gaete, Verónica. (2015). Desarrollo psicosocial del adolescente. Revista chilena de pediatría, 86 (6), 436-443. https://dx.doi.org/10.1016/j.rchipe.2015.07.005

Goleman, D. (1995). What's your emotional intelligence quotient? You'll soon find out. Utne Reader, November/December.

Goleman, D. (1996). La inteligencia emocional. Kairo.

Gómez, J. M., Galiana, D., \& León, D. (2000). Que debes saber para mejorar tu empleabilidad. Universidad Miguel Hernández. Published.

Hernandez S., R., Fernández C., C., \& Baptista L., M. (2014). Metodología de la investigación. McGraw-Hill Interamericana.

Rojas A., M. (2018). Estrés académico y estrategias de afrontamiento en estudiantes de la escuela de enfermería Padre Luis Tezza. Universidad Nacional de Educación Enrique Guzmán y Valle Alma Máster del Magisterio Nacional. Published.

Universidad Autónoma del Estado de Hidalgo. (2019). Anuario Estadístico 2018. UAEH. 